www.jmscr.igmpublication.org

Impact Factor 5.84

Index Copernicus Value: 71.58

ISSN (e)-2347-176x ISSN (p) 2455-0450

crossref DOI: _https://dx.doi.org/10.18535/jmscr/v5i11.13

Journal Of Medical Science And Clinical Research

IGM Publication

An Official Publication of IGM Publication

\title{
Structural Asymmetries of inferior Triangular Gyri, Heschl'S Gyri and Globus Pallidi in Children- A Voxel Based Morphometric Assessment of Primary and Secondary Language Areas in Children
}

\author{
Authors \\ Dr Ruma Madhu Sreedharan, Dr Sheela Kumari R, Dr Sanjeev V Thomas \\ GMC, Trivandrum
}

\begin{abstract}
Background and Purpose: The objective was to assess language lateralization based on the volumetric analysis involving Inferior Triangular Gyrus (ITG),Inferior Opercular Gyrus (IOG), Superior Temporal Gyrus (STG), and secondary language areas like Heschl's Gyrus (HG), Hippocampus, Globus Pallidi (GP), Putamen and Thalamus.

Methods: Thirty six right handed children with normal brain MRI were selected for the study. Using Voxel Based Morphometry (VBM) structural volumetric differences between ITG, IOG, STG, HG, Hippocampus and thalami of right and left hemispheres were assessed by VBM, an automated technique that identifies differences in the local gray matter volume between groups using high-resolution Tl-weighted MRI.

Results: There was significant increase in the volume of left GP compared to the right GP $(<0.001)$, left ITG compared to right side ( $p<0.03)$, left Heschl's Gyrus compared to the right side ( $p<0.05)$ while the mean grey matter volume of right put a men and right IOG was larger compared to the left with a $p$ value of 0.04 and 0.007 respectively. No significant differences in the mean grey matter volume was seen between right and left hippocampus, thalami and STG ( $p=0.30,0.795$ and 0.175 respectively).

Conclusion: Our study highlights the importance of left ITG,HG and GP in language processing.

Keywords: Voxel based Morphometry, inferior triangular gyrus, heschl's gyrus, globus pallidum.
\end{abstract}

\section{Introduction}

Cerebral lateralization refers to the tendency of the brain to have some neuronal functions predominant in one hemisphere. Although the human brain has two separate hemispheres which appear identical macroscopically, they have distinct cognitive, language and memory functions.

Lateralization of brainis different from specialization as each human`s brain develop differently leading to the unique lateralization in individuals. One of the first break through in the history of lateralization was the discovery of Broca's and Wernicke's areas. The concept of language lateralization (LL) was first introduced by Paul Broca and Carl Wernicke as early as the nineteenth century ${ }^{(1,2)}$. The posterior part of the inferior frontal gyrus-Broca's area (BA) is involved in speech production while the posterior part of the superior temporal gyrus Wernicke's area (WA) controls speech comprehension. Many tests like the Wada test, positron-emission tomography (PET), and electro cortical stimulation mapping are available to study $\mathrm{LL}^{(3)}$. Though the Wada test is the most widely used test, it is invasive, expensive, and provides information of only language lateralization and not specific language localization to any part of the lobes. It`s a difficult test to carry out in young children. One of the most lateralized brain functions is language. Language areas in the brain 
are asymmetrical as FMRI had shown that $95 \%$ of right-handed and $60-75 \%$ of left-handed individuals have left hemispheric lateralization. Functional MRI (FMRI) is a novel imaging tool used for studying LL. It measures changes in blood oxygenation level which produces $\mathrm{T} 2 *$ signal intensity changes that can be easily detected and quantified (4). But it cannot be done in uncooperative subjects, patients with terminal neurodegenerative disorders, in young children and psychiatric patients as it requires active participation. Morphometry methodology is an automated technique that investigates overall differences in brain volume by a normalization process using the statistical approach known as statistical parametric mapping (SPM) and is least subject to individual variation ${ }^{(5)}$. It involves a voxel-wise statistical comparison of the local concentration of gray matter within or between two groups of subjects. To ascertain whether functional asymmetry is related to structural asymmetry, a VBM study was conducted in 36healthy right handed children in the age group 8-12 years to see if there are any volumetric differences between right and left hemispheres with regard to language. VBM of language areas like inferior triangular gyrus (ITG), Inferior opercular gyrus (IOG), superior temporal gyrus (STG), and secondary language areas like heschl's gyrus (HG), hippocampus, globus pallidi (GP), putamen and thalamus were studied.

The advantages of VBM in children are that it is noninvasive, has no radiation exposure, and can be repeated and doesn't require subject cooperation or attention. It can be done in mentally challenged individuals also. The study was done to explore structural correlates of language function by comparing the brain volumetry of both cerebral hemispheres of healthy children. Our hypothesis was like FMRI, VBM can also demonstrate lateralization of language areas.

\section{Materials \&Methods}

\section{Study Design and study population}

Study design is cross sectional analytic study. Thirty six children in the age group 8-12 years were selected. They had no past history of neurological or psychiatric diseases, and no contraindications of neuroimaging. Exclusion criteria included children with severe mental retardation, history of birth asphyxia, epilepsy, history of any acquired neurological insult, congenital malformation or metallic implants. All Children could speak Malayalam language, had normal vision, hearing, neurological examination and normal structural MRI. The study was approved by the Institutional Ethics committee and written informed consent from parents and assent forms from children were taken. Only right handed individuals were included in the project.

Magnetic Resonance Imaging Protocol and analysis

The MR Imaging was performed in 1.5 Tesla Magnetic Resonance scanner (Avanto SQ engine, Siemens, Erlangen, Germany). For VBM a 3-D FLASH sequence (Fast Low Angle Shot) which is a high resolution 3D T1 weighted images of brain was obtained (TR/TE 11/4.94 ms, flip angle $15^{\circ}$, FOV $256 \mathrm{~mm}$, slice thickness $1 \mathrm{~mm}$ and matrix of $256 \times 256$ ). During MRI head coil with soft pads placed around the head to immobilize the head. All children were given proper training for reducing movement artefacts during the scan period.

\section{VBM data analysis}

\section{First level Analysis}

VBM data analysis was done using VBM8 tool (8 (VBM8; http://dbm.neuro.uni-jena.de/vbm/) in SPM8 - Statistical Parametric Mapping software (Wellcome Department of Imaging

Neuroscience, University College. London. www.fil.ion.ucl.ac.uk/spm) in MATLAB 7.7

(Math Works, Natwick MA, USA) according to the standard protocol.

\section{Creation of pediatric template}

First an age and sex matched pediatric template was generated using TOM8 tool box, which is an extension software of SPM 8.(Figure1)It's made from the reference data from the NIH study of 
normal brain development after incorporating age, gender and handedness using 'average pairs approach. From our data we created the template as there is difference in the structure and plasticity between pediatric and adult brain. ${ }^{(6)}$

\section{VBM steps}

Basic steps in VBM pre-processing include spatial normalization, segmentation, modulation, and Smoothing. (Figure 2)

The structural T1weighted images were first aligned properly using antero-posterior commissure line and then segmented into grey matter (GM), white matter (WM) and cerebrospinal fluid (CSF) using tissue probability maps. The images were normalized to the template. A deionizing filter is applied to remove noise and the segmented GM, WM and CSF images were modulated and normalized using affine and nonlinear method. The normalized modulated non-linear images were checked for sample homogeneity and one outlier image was detected and hence removed from final analysis. Using a $8 \times 8 \times 8 \mathrm{~mm} 3$ FWHM Gaussian filter the images were smoothened and finally checked for their spatial normalization using Check Reg facility in SPM window.

\section{Segmentation Analysis}

Segmentation was also done using VBM 8 toolbox. The volumes of ITG,IOGSTG , Hippocampus, transverse temporal gyri (also called Heschl's gyrus-HG), GP, putamen and thalami were computed for both sides and compared.Volumes of selected regions of interest were measured using an approach based on Automated Anatomical Labeling (AALAnatomical Automatic Labeling; http://www.cyceron.fr/web/aal), which is a digital atlas of the human brain.

The normalized images obtained after preprocessing were then segmented into GM, WM, and CSF using the customized prior probability maps. The modulated gray matter images were then smoothed with an $8 \mathrm{~mm}$ isotropic Gaussian kernel. Individual grey matter structures were segmented out based on AAL atlas using segmentation algorithm. Final output was read in MATLAM window.

\section{Statistical Analysis}

Statistical analysis was done with SPSS software Statistical Package for Social Science Version 20 for windows (SPSS 20,SPSS Inc. Chicago, Illinois, USA). The quantitative variables were compared by student $t$ test and. Significance level was set at $p$ value $<0.05$.

\section{Ethics}

The study protocol was approved by Institutional Ethical Committee. Written informed consent from the parents and assent forms from the children were obtained.

\section{Results}

There were 36 children ( 24 males) who fulfilled selection criteria and consented to participate in the study. The mean age was $10.4 \pm 0.94$ (mean \pm $\mathrm{SD})$

\section{VBM Results}

The mean grey matter volume of left and right ITG, IOG, STG were 9.24 \pm 2.11 and $5.33 \pm 1.34$, $3.68 \pm 0.81$ and $4.02 \pm 0.88,9.35 \pm 2.25$ and 9.94 \pm 2.57 respectively. The mean grey matter volume of left and right $\mathrm{HG}$, hippocampi, GP, putamen and thalami were $4.32 \pm 1.34$ and $3.62 \pm 0.89,3.24$ \pm 0.78 and $3.09 \pm 0.62,3.46 \pm 0.56$ and 3.51 $\pm 1.02,3.62 \pm 0.56$ and $4.11 \pm 0.98$ respectively.

The Inferior triangular gyrus (ITG), Heschl`s Gyrus (HG), Globus Pallidus (GP)were significantly left lateralized compared to the right side. (Figure 3)

There was significant increase in the volume of left GP compared to the right GP $(<0.001)$, left ITG compared to right side ( $\mathrm{p}<0.03)$, left Heschl`s Gyrus compared to the right side $(\mathrm{p}<0.05)$ while the mean grey matter volume of right putamen and right IOG was larger $(\mathrm{p}<0.04)$ compared to the left with a $p$ value of 0.04 and 0.007 respectively. No significant differences in the mean grey matter volume was seen between right and left hippocampus, thalami and STG ( $\mathrm{P}=0.30$, 0.795 and 0.175 respectively). 
Figure 1. Pediatric template
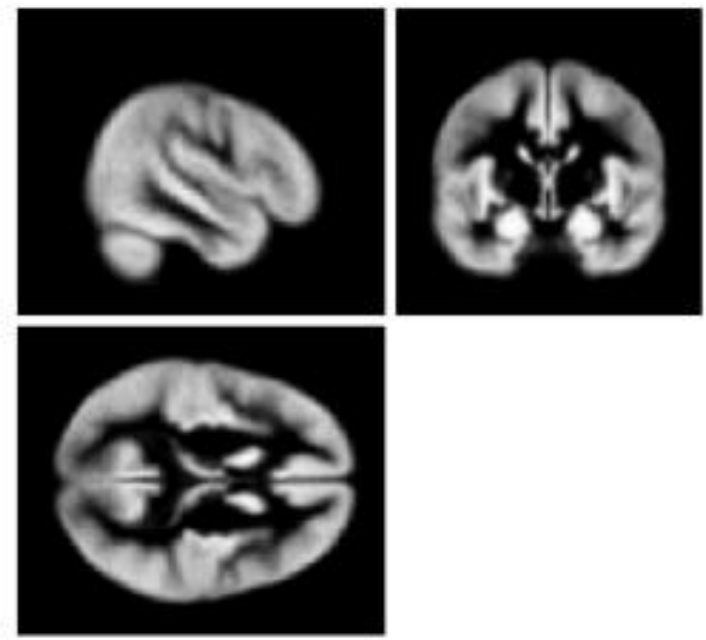

Figure 2. VBM processing pipeline

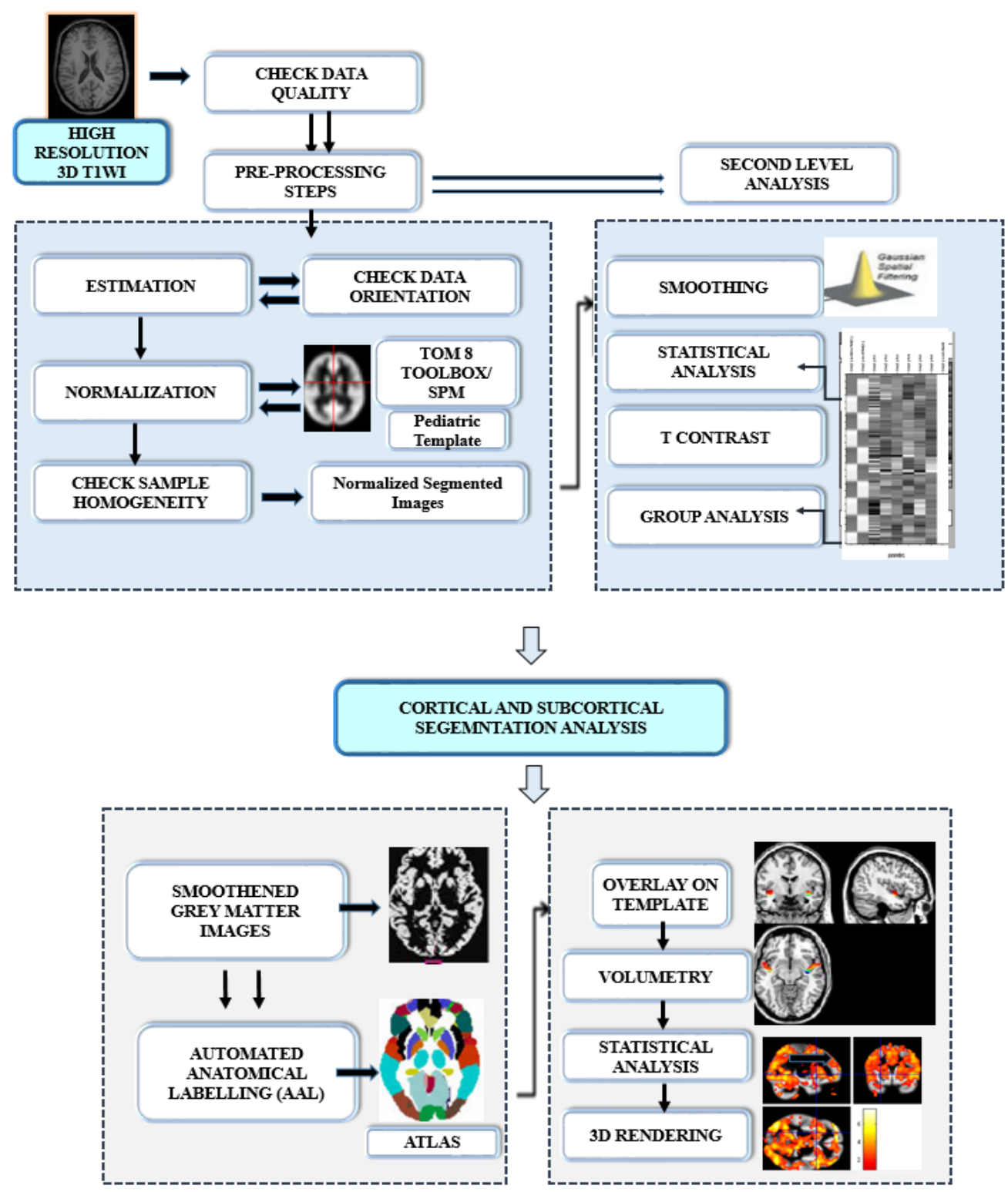


Figure 3a. Bar diagram showing volume of left Inferior Triangular Gyrus (ITG), Inferior opercular gyrus (IOG), superior temporal gyrus (STG), and heschl's gyrus (HG).
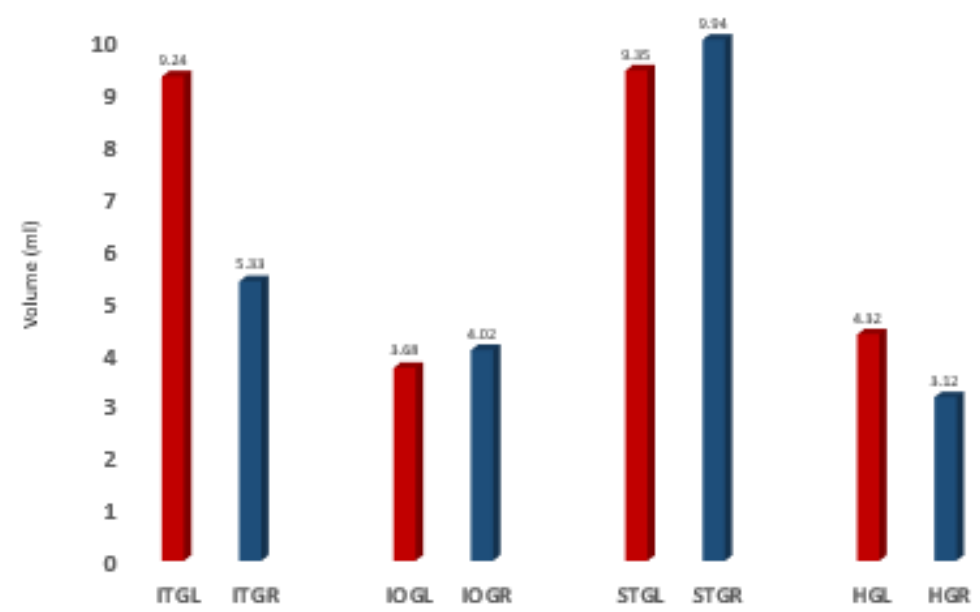

Figure 3b. Bar diagram showing volumes of hippocampus, globus pallidi, putamen and thalamus.

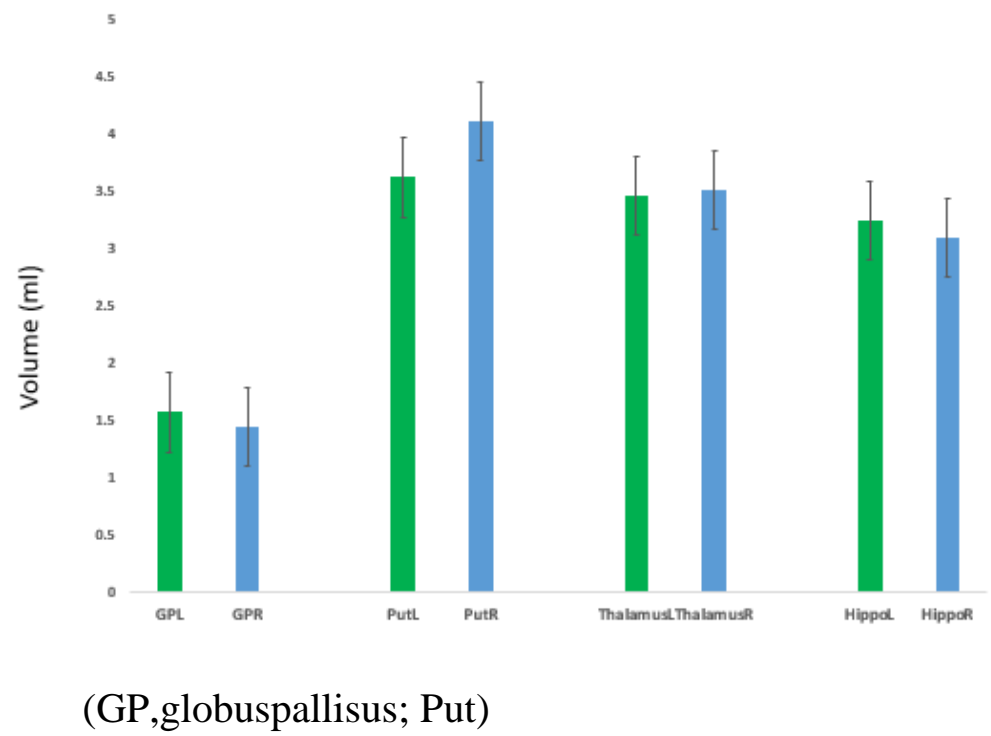

\section{Discussion}

Though cerebral hemispheres are macroscopically similar, they differ in the function and in most humans the left hemisphere is dominant for language, whereas the right one is dominant for visuo-spatial functions. The present study explored structural correlates of functional language dominance by comparing VBM in healthy children to study volumetric measurements of cortical and deep grey matter structures involved in language for assessing language lateralization. Though originally devised to examine structural abnormalities in patients, the technique can also be used with healthy subjects. Our objective was to see if there wasany lateralization of volume of superficial and deep grey matter structures related to language. We got left lateralization of ITG, HG and GP compared to the right hemisphere, which may point to hemispheric language lateralization.

The importance of frontal lobe and language processing was known as early as mid-nineteenth century when Paul Broca first described the importance of left ITG in language processing. He 
studied 8 right handed individuals with left hemispheric lesions who lost the ability of speech and concluded the importance of left prefrontal region in speech. ${ }^{(1)}$ Broca's motor speech area is located in the ventral-posterior part of the inferior frontal gyrus with a rostral part namely pars triangularis and a caudal part called pars opercularis. Though histologically pars opercularishas a scantygranular area, it is considered to be a part of motor territory while pars triangularis is a granular area which has the same histological architecture as other brain regions situated in the prefrontal cortex. ${ }^{(7,8)}$ Our study showing lateralization of left ITG also highlights the importance of left frontal language areas.

Functional magnetic resonance imaging (FMRI) is relatively novel noninvasive technique for language mapping based on monitoring regional changes in bloodoxygenation resulting from neural activity. ${ }^{(9,10)}$ FMRI studies have also shown that Left Inferior frontal cortex ${ }^{(11-13)}$ has been involved in language comprehension as well as other linguistic functions like phonology, morphology and semantics. ${ }^{(14)}$ Bindher et al studied FMRI in 30 right handed individuals using phonetic and semantic task and showed activation in the frontal, temporal, and parietal lobes. ${ }^{(15)} \mathrm{A}$ longitudinal FMRI study of language development in children aged 5-11 years ${ }^{(16)}$ demonstrated that with increasing age, there is progressive participation in language processing areas and include inferior/middle frontal, middle temporal and angular gyri of the left hemisphere. Now pars triangularis is also considered to have an important role in attention, inhibition, verbal working memory and hence considered an important functional language network hub. ${ }^{(17)}$ Many other studies have associated cortical grey matter asymmetries with language function. But only few VBM studies with regard to language lateralization are available. Dorsaint-Pierre et al and Foundas et al had VBM study in epileptic brain and showed leftward asymmetry of pars triangularis. ${ }^{(14,18)}$
The transverse temporal gyri or heschl's gyri (BA $41 \& 42$ ) are seen within the lateral sulcus in primary auditory cortex and run medio-laterally towards the center of the brain. Asymmetries of HG are well known in literature. ${ }^{(18,19)}$ HG duplication pattern was found to be a significant factor affecting the surface area of the most posterior part of PT and its asymmetry, which inturn was specifically associated with variability in verbal performances. ${ }^{(20)}$ HG gyrification pattern may also point towards an important structural correlates of language processing. Left-handers` HG surface asymmetries were found to be significantly different from those of right-handers with gyrification patterns producing an effect on HG surface and asymmetry. ${ }^{(21)}$ The decreased lateralization of $\mathrm{HG}$ duplications and anterior $\mathrm{HG}$ asymmetry in left-handed individuals high lights the importance of the HG gyrification patterns as a potential biomarker of speech lateralization. The present study of VBM also showed significant left lateralization of volume of HG though we didn't study the gyrification pattern and its asymmetry. Though basal ganglia was initially considered to be an integral part of motor control and coordination, it has now well accepted role in language processing. ${ }^{(22-24)}$ Patients with left basal ganglia lesions presented with agrammatic sentences and lexical semantic mistakes suggesting an important role in regulation of speech production and lexical and semantic language processing. ${ }^{(22)}$ Clinical trials have shown many Parkinson`s Disease patients presenting with language problems thus highlighting a definitive role of basal ganglia in language comprehension but the actual role is still debated. Our study showing lateralization of GP but not putamen which may be unrelated to lateralization but need to be augmented with studies using a larger cohort. No significant lateralization was seen with temporal or parietal language areas or thalami or hippocampi.

The main strength of the study was subject selection. The study had the presence of fairly good number of participants. Only few VBM 
studies have been conducted in normal children. In the present study the subjects were selected based on their language dominance by handedness as in many other lateralization studies. Only healthy individuals with normal brain MRI were selected while many VBM studies involving language were computed in epilepsy patients. The data was analyzed by VBM which is an automated method for measuring grey matter differences across subjects and less sensitive to bias compared to the traditional ROI-based approaches. We used a standard pediatric template made from the representation of our sample data for overlying our results. The data was smoothened with $8 \mathrm{~mm}$ Gaussian Kernel for reducing noise.

The study had several limitations. First, left handed subjects were not recruited and hence we couldn`t study atypical language lateralization. FMRI was not used for calculating handedness and our VBM results were not compared with FMRI activation pattern to assess the full spectrum of functional structural asymmetry. And due to the limited sample size the interactions between handedness, gender, and hemispheric dominance were not studied. So future studies with larger sample sizes might tackle this question by including handedness as a separate factor in the statistical analysis. Such studies can be of particular use for assessing and comparing subjects with atypical language lateralization. Further studies by combining functional imaging (FMRI) with structural imaging of grey (VBM) and white matter (diffusion tensor imaging) may complement our results and help in underpinning neuronal correlates of language lateralization.

\section{References}

1. Sur le siège de la faculté du langage articulé - 1865 Broca.pdf [Internet]. [cited 2017 Jul 21]. Available from: http://homes.mpimf-

heidelberg.mpg.de/ mhelmsta/pdf/1865\% 20Broca.pdf

2. Carl Wernicke. Der aphasische Symptomencomplex: Eine psychologische
Studie auf anatomischer Basis [Internet]. Cohn; 1874 [cited 2017 Jul 21]. 80 p. Available from: http://archive.org/details/deraphasischesy0 0werngoog

3. Wada J, Rasmussen $\mathrm{T}$. Intracarotid Injection of Sodium Amytal for the Lateralization of Cerebral Speech Dominance. Collections. 2009 May 5;112(2):266-82.

4. Binder JR, Swanson SJ, Hammeke TA, Morris GL, Mueller WM, Fischer M, et al. Determination of language dominance using functional MRI: a comparison with the Wada test. Neurology. 1996 Apr;46(4):978-84.

5. Ashburner J, Friston KJ. Voxel-based morphometry--the methods. NeuroImage. 2000 Jun;11(6 Pt 1):805-21.

6. Wilke M, Holland SK, Altaye M, Gaser C. Template-O-Matic: a toolbox for creating customized pediatric templates. NeuroImage. 2008 Jul 1;41(3):903-13.

7. Amunts $\mathrm{K}$, Schleicher $\mathrm{A}$, Bürgel $\mathrm{U}$, Mohlberg $\mathrm{H}$, Uylings HB, Zilles $\mathrm{K}$. Broca's region revisited: cytoarchitecture and intersubject variability. J Comp Neurol. 1999 Sep 20;412(2):319-41.

8. Anwander A, Tittgemeyer M, von Cramon DY, Friederici AD, Knösche TR. Connectivity-Based Parcellation of Broca's Area. Cereb Cortex N Y N 1991. 2007 Apr;17(4):816-25.

9. Ogawa S, Lee TM, Kay AR, Tank DW. Brain magnetic resonance imaging with contrast dependent on blood oxygenation. Proc Natl Acad Sci U S A. 1990 Dec;87(24):9868-72.

10. Ogawa S, Menon RS, Tank DW, Kim SG, Merkle H, Ellermann JM, et al. Functional brain mapping by blood oxygenation leveldependent contrast magnetic resonance imaging. A comparison of signal characteristics with a biophysical model. Biophys J. 1993 Mar;64(3):803-12. 
11. Tyler LK, Marslen-Wilson WD, Randall B, Wright P, Devereux BJ, Zhuang J, et al. Left inferior frontal cortex and syntax: function, structure and behaviour in patients with left hemisphere damage. Brain J Neurol. 2011 Feb;134(Pt 2):41531.

12. Gabrieli JD, Poldrack RA, Desmond JE. The role of left prefrontal cortex in language and memory. Proc Natl Acad Sci U S A. 1998 Feb 3;95(3):906-13.

13. Burton MW. The role of inferior frontal cortex in phonological processing. Cogn Sci. 2001 Sep 1;25(5):695-709.

14. Foundas AL, Leonard CM, Gilmore RL, Fennell EB, Heilman KM. Pars triangularis asymmetry and language dominance. Proc Natl Acad Sci U S A. 1996 Jan 23;93(2):719-22.

15. Binder JR, Frost JA, Hammeke TA, Cox RW, Rao SM, Prieto T. Human Brain Language Areas Identified by Functional Magnetic Resonance Imaging. J Neurosci. 1997 Jan 1;17(1):353-62.

16. Szaflarski JP, Schmithorst VJ, Altaye M, Byars AW, Ret J, Plante E, et al. A longitudinal fMRI study of language development in children age 5-11. Ann Neurol. 2006 May;59(5):796-807.

17. Caplan D, Waters GS. Verbal working memory and sentence comprehension. Behav Brain Sci. 1999 Feb;22(1):77-94126.

18. Dorsaint-Pierre R, Penhune VB, Watkins KE, Neelin P, Lerch JP, Bouffard M, et al. Asymmetries of the planum temporale and Heschl's gyrus: relationship to language lateralization. Brain J Neurol. 2006 May;129(Pt 5):1164-76.

19. Schneider P, Sluming V, Roberts N, Scherg M, Goebel R, Specht HJ, et al. Structural and functional asymmetry of lateral Heschl's gyrus reflects pitch perception preference. Nat Neurosci. 2005 Sep;8(9):1241-7.

20. Tzourio-Mazoyer N, Mazoyer B. Variations of planum temporale asymmetries with Heschl's Gyri duplications and association with cognitive abilities: MRI investigation of 428 healthy volunteers. Brain Struct Funct. 2017 Aug;222(6):2711-26.

21. Marie D, Jobard G, Crivello F, Perchey G, Petit L, Mellet E, et al. Descriptive anatomy of Heschl's gyri in 430 healthy volunteers, including 198 left-handers. Brain Struct Funct. 2015 Mar;220(2):72943.

22. Fabbro F, Clarici A, Bava A. Effects of left basal ganglia lesions on language production. Percept Mot Skills. 1996 Jun;82(3 Pt 2):1291-8.

23. Wildgruber D, Ackermann H, Grodd W. Differential Contributions of Motor Cortex, Basal Ganglia, and Cerebellum to Speech Motor Control: Effects of Syllable Repetition Rate Evaluated by fMRI. NeuroImage. 2001 Jan;13(1):101-9.

24. Zenon A, Olivier E. Contribution of the basal ganglia to spoken language: Is speech production like the other motor skills? Behav Brain Sci. 2014 Dec;37(6):576. 